\title{
Reconstruction of Signal from Samples of Its Integral in Spline Subspaces ${ }^{\star}$
}

\author{
Jun Xian, Yongjin Li, and Wei Lin ${ }^{\star \star}$ \\ Department of Mathematics, Sun Yat-sen University, Guangzhou, 510275, China
}

\begin{abstract}
In this paper, we study the reconstructing of signals based on regular and irregular incremental integral samples in some nonbandlimited space--spline subspace, and we obtain reconstructing formulas with a new method.
\end{abstract}

\section{Introduction}

In digital signal and image processing, digital communication, etc., a continuous signal is usually represented and processed by using its discrete samples. For a bandlimited signal of finite energy, it is completely characterized by its samples, by the famous classical Shannon sampling theorem. However, in many real applications sampling points are not always regularly and sampled value may not be values of a signal $f$ precisely at times $x_{k}$ for the inertia of the measurement aparatus. As for the signal spaces, they are not always bandlimited signal of finite energy. The problem arose initially in the design of an interferometer in which the interferogram is obtained using a continuously moving mirror, but may also have bearing in other problem in which the data are smoothed by an integrating sensor, such as CCD array with slow response time compared to the sample integral. So we need to give the reconstruction of signal from samples of its integral. In this paper, we discuss the reconstruction of signal from samples of its integral in spline subspaces.

Though Sun and Zhou studied this problem in [7], their result and method are not easy to apply in practical application and computation. We will give reconstruction formula from samples of its integral in spline subspaces with the simple method. And the reconstruction formula is more practical in application.

\section{Reconstruction of Signal from Regular Incremental Integral Samples in Spline Subspaces}

Let $y_{k}=\int_{-\infty}^{k+1} f(t) d t, y_{k-1}=\int_{-\infty}^{k} f(t) d t, z_{k}=y_{k}-y_{k-1}$. We refer to $\left\{y_{k}\right\}$ as the set of cumulative integral samples. The problem is to reconstruct the signal $f$

* This work is supported in part by the China-NSF, the Guangdong-NSF and the Foundation of Sun Yat-Sen University Advanced Research Centre.

** E-mail address: xianjun11@sina.com (Jun Xian), stslw@zsu.edu.cn (Wei Lin), stslyj@zsu.edu.cn (Yongjin Li) 
from $\left\{z_{k}\right\}$ (incremental integral samples). We now introduce some notations and lemmas used in Section 2 and Section 3. In this paper, the Fourier transform of $f \in L^{2}(\mathbb{R})$ is defined by $\hat{f}(\omega)=\int_{\mathbb{R}} f(x) e^{-i x \omega} d x . V_{N}=\left\{\sum_{k \in Z} c_{k} \varphi_{N}(\cdot-k):\left\{c_{k}\right\} \in\right.$ $\left.\ell^{2}\right\}$ is spline subspace generated by $\varphi_{N}=\chi_{[0,1]} * \cdots * \chi_{[0,1]}$ (N convolutions), $N \geq 1$.

Lemma 2.1 ${ }^{[5]}\left\{\varphi_{N}(\cdot-k): k \in Z\right\}$ is Riesz basis for $V_{N}, A_{N}=\sum_{k} \mid \hat{\varphi}_{N}(\pi+$ $2 k \pi)\left.\right|^{2}$ and $B_{N}=1$ are its lower and upper bounds, respectively.

Lemma 2.2 ${ }^{[2]}$ If $\left\{x_{n}\right\}$ is real sequence with $\sup _{i}\left(x_{i+1}-x_{i}\right)=\delta<1$, then there exist constants $c$ and $C$ such that for arbitrary $f \in V_{N}$ we have $c\|f\|_{2}^{2} \leq$ $\sum_{i}\left|f\left(x_{i}\right)\right|^{2} \leq C\|f\|_{2}^{2}$.

Lemma 2.3 Suppose that $\hat{\tilde{\varphi}}_{N}(\omega)=\frac{\hat{\varphi}_{N}(\omega)}{\left|\Pi_{N}\left(e^{-i \omega}\right)\right|}$, where $\Pi_{N}(\omega)=$ $\sum_{n=0}^{2 N-2} \varphi_{2 N}(n+1) \omega^{k}$. Then $k_{x}(\omega)=k(\omega, x)=\sum_{n} \tilde{\varphi}_{N}(w-n) \overline{\varphi_{N}(x-n)}$ is reproducing kernel for $V_{N}$.

Theorem 2.1 ${ }^{[7]}$ For arbitrary $f \in V_{N}$, we have

$$
f(x)=\sum_{k \in Z} f\left(k+\frac{N+1}{2}\right) S(x-k),
$$

where $\hat{S}(\omega)=\frac{\hat{\varphi}_{N}(\omega)}{\sum \varphi_{N}\left(k+\frac{N+1}{2}\right) e^{-i k \omega}}$ and $V_{N}$ is spline subspaces generated by $\varphi_{N}=$ $\chi_{[0,1]} * \cdots * \chi_{[0,1]}(N \geq 1$ convolutions $)$.

Lemma 2.4 Let $y(t)=\int_{-\infty}^{t} f(x) d x$ and $f \in V_{N}$, then $y \in V_{N+1}$.

By Theorem 2.1 and Lemma 2.4, we have the following Theorem 2.2.

Theorem 2.2 Let

$$
y_{k}=\int_{-\infty}^{k+\frac{1}{2}+1} f(t) d t, y_{k-1}=\int_{-\infty}^{k+\frac{1}{2}} f(t) d t, z_{k}=y_{k}-y_{k-1}
$$

Then for any $f \in V_{N}$, we have reconstruction formula

$$
f(t)=\sum_{k \in Z} z_{k} h(t-k)
$$

where $h$ is defined by

$$
h_{k}=h_{1 k}+h_{k+1}, \hat{h}_{1}(\omega)=i \pi \omega \frac{\hat{\varphi}_{N+1}(\omega)}{\sum \varphi_{N+1}\left(k+\frac{N}{2}+1\right) e^{-i k \omega}},
$$

$h_{k}(\cdot)=h(\cdot-k)$ and $h_{1 k}(\cdot)=h_{1}(\cdot-k)$.

Proof. From Lemma 2.4, we know $y \in V_{N+1}$. Then we use Theorem 2.1 to obtain

$$
y(t)=\sum_{k \in Z} y\left(k+\frac{N}{2}+1\right) S_{1}(t-k)
$$


where $\hat{S}_{1}(\omega)=\frac{\hat{\varphi}_{N+1}(\omega)}{\sum_{k} \varphi_{N+1}\left(k+\frac{N}{2}+1\right) e^{-i k \omega}}$. Through derivation calculus are acted in both sides of equality $(*)$, we can imply

$$
f(t)=\sum_{k \in Z} y_{k} S_{1}^{\prime}(t-k)=\sum_{k \in Z} y_{k} h_{1}(t-k)=\sum_{k \in Z} z_{k} h(t-k) .
$$

The above last equality derives from the assumption $h_{k}=h_{1 k}+h_{k+1}$.

Remark 1. a.Actually, Theorem 2.2 show the reconstruction formula from regular incremental integral samples in $V_{N}$.

b. The above reconstruction formula can be rewritten as the following:

$$
f(t)=\sum_{k \in Z} \int_{k+\frac{N}{2}+1}^{k+\frac{N}{2}} f(x) d x h(t-k)=\sum_{k \in Z}\langle f, u(\cdot-k)\rangle h(t-k),
$$

where $u(\cdot)=\chi_{\left[\frac{N}{2}, \frac{N}{2}+1\right]}(\cdot)$. This induces that incremental integral samples can be regarded as a special weighted samples.

c. The interpolation function $h$ is practical in application.

\section{Reconstruction of Signal from Irregular Incremental Integral Samples on Spline Subspace}

Next, we will study the reconstruction from irregular incremental integral samples. We will show the following Theorem 3.1 before we giving the main result.

Theorem 3.1 Let sampling set $X=\left\{x_{n}\right\}$ be a real sequence and satisfy $\sup _{i}\left(x_{i+1}-x_{i}\right)=\delta<1$. Then $\left\{k\left(\cdot, x_{j}\right)\right\}$ is frame for $V_{N+1}$ and there exist dual frames $\left\{\tilde{k}_{x_{j}}: x_{j} \in X\right\} \subset V_{N+1}$ such that for any $f \in V_{N+1}$, we have

$$
f(x)=\sum_{j \in Z}\left\langle f, k_{x_{j}}\right\rangle \tilde{k}_{x_{j}}(x)=\sum_{j \in Z} f\left(x_{j}\right) \tilde{k}_{x_{j}}(x)
$$

where $k(\cdot, \cdot)$ as the same in Lemma 2.3.

Proof. From the discussion of [1, 3], we know Theorem 3.1 holds.

We make little changes for the proof of Theorem 2.2 to obtain the proof of the following Theorem 3.2. So we will omit its proof.

Theorem 3.2 Let

$$
y_{k}=\int_{-\infty}^{x_{k}} f(t) d t, y_{k-1}=\int_{-\infty}^{x_{k-1}} f(t) d t, z_{k}=y_{k}-y_{k-1}
$$


and sampling set $X=\left\{x_{n}\right\}$ is real sequence with $\sup _{i}\left(x_{i+1}-x_{i}\right)=\delta<1$. Then for any $f \in V_{N}$ we have reconstruction formula

$$
f(t)=\sum_{k \in Z} z_{k} \tilde{h}_{x_{k}}(x),
$$

where $\left\{\tilde{h}_{x_{k}}\right\}$ is given by $h_{x_{j}}=\tilde{h}_{x_{j}}-\tilde{h}_{x_{j+1}}(j \in Z), h_{x_{j}}(x)=\tilde{k}_{x_{j}}^{\prime}(x)$ is derivative of $\tilde{k}_{x_{j}}(x)$ and $\tilde{k}_{x_{j}}(x)$ as the same in Theorem 3.1 .

\section{Conclusion}

In this paper, we obtain reconstruction formula from samples of its integral in spline subspaces with the simple new method. And the reconstruction formula is more practical in application. Furthermore, we obtain reconstruction formula of signal from irregular incremental integral samples in spline subspace too. We conjecture the new method remains validity for a much large class of shiftinvariant spaces. We will study it in future work.

\section{References}

1. Aldroubi, A.: Non-uniform weighted average sampling and reconstruction in shiftinvariant and wavelet spaces, Appl. Comput. Harmon. Anal 13(2002)156-161.

2. Aldroubi, A., K. Gröchenig.: Beurling-Landau-type theorem for non-uniform sampling in shift invariant spline spaces. J. Fourier. Anal. Appl, 6(2000)93-103.

3. Aldroubi, A., K. Gröchenig.: Non-uniform sampling and reconstruction in shiftinvariant spaces., SIAM Rev 43(2001)585-620.

4. Aldroubi, A., Unser, M., Eden, M.: Cardinal spline filters: Stability and convergence to the ideal sinc interpolator," Singal. Processing 28(1992)127-138.

5. Chui, C. K.: An introduction to Wavelet, Academic Press, New York,1992

6. Liu, Y.: Irregular sampling for spline wavelet subspaces, IEEE Trans. Inform. Theory 42(1996)623-627.

7. Sun, W. C., Zhou., X. W.: Average sampling in spline subspaces. Appl. Math. Letter, 15(2002)233-237.

8. Sun, W. C., Zhou, X. W.: Reconstruction of bandlimited signals from local averages. IEEE Trans. Inform. Theory, 48(2002)2955-2963.

9. Wang, J.: Spline wavelets in numerical resolution of partial differential equations, International Conference on Wavelet Analysis and its application, AMS/IP Studies in Advanced Mathematics, 25(2002)257-276.

10. Xian, J., Lin, W.: Sampling and reconstruction in time-warped spaces and their applications. to appear in Appl. Math. Comput, 2004. 